\title{
DENSE PIXEL MATCHING BETWEEN UNRECTIFIED AND DISTORTED IMAGES USING DYNAMIC PROGRAMMING
}

\author{
Jerome Thevenon, Jesus Martinez-del-Rincon, Romain Dieny and Jean-Christophe Nebel \\ Digital Imaging Research Centre, Kingston University, Penhryn Road, \\ Kingston-Upon-Thames, KT1 2EE, Surrey, UK \\ \{J.Thevenon, Jesus.Martinezdelrincon, R.Dieny, J.Nebel\}@kingston.ac.uk
}

\begin{abstract}
Keywords: Stereo correspondence, dynamic programming, unrectified images, distorted images.
Abstract: In this paper, a novel framework for dense pixel matching based on dynamic programming is introduced. Unlike most techniques proposed in the literature, our approach assumes neither known camera geometry nor the availability of rectified images. Under such conditions, the matching task cannot be reduced to finding correspondences between a pair of scanlines. We propose to extend existing dynamic programming methodologies to a larger dimensional space by using a 3D scoring matrix so that correspondences between a line and a whole image can be calculated. After assessing our framework on a standard evaluation dataset of rectified stereo images, experiments are conducted on unrectified and non-linearly distorted images. Results validate our new approach and reveal the versatility of our algorithm.
\end{abstract}

\section{INTRODUCTION}

Dense pixel matching is a low-level process which is involved in many computer vision applications including navigation, security, entertainment and video surveillance. Not only is it often an essential step in 3D reconstruction from a pair of stereo images, but it is also used in object detection and video tracking, especially when the camera is not fixed (Note, et al., 2006) (Yaguchi,, et al., 2009) as it is the case with camera phones (Yin, et al., 2007).

Recent reviews describe and analyse the many algorithms which have been proposed to address this matching process (Scharstein \& Szeliski, 2002) (Lazaros, et al., 2008). Most approaches performing dense pixel matching assume images have been rectified so that their task can be reduced to finding correspondences between a pair of scanlines. Among these techniques, those based on dynamic programming (DP) are of particular interest since they combine good accuracy with low computational complexity as demonstrated by a recent real-time FPGA hardware implementation (MacLean, et al., 2010). Their main drawback is that they require the knowledge to project images onto a common coordinate system. Although, in many applications those transformations can be estimated by either camera calibration or image rectification, calibration is sometimes either impossible or impractical, whereas the computational cost of accurate rectification models prohibits their usage in realtime application. Moreover, none of these methods is suitable when a camera lens displays unexpected distortions, such as those generated by raindrops, weatherproof covers or dust.

In this paper, we propose a novel DP-based dense pixel matching algorithm which can handle unrectified and non-linearly distorted images. After a state-of-the-art review, we detail our novel matching algorithm. Finally, it is validated with experiments conducted on unrectified images and images displaying significant deformations.

\subsection{Related Work}

Generally, pixel matching approaches (Barnard, et al., 1982) (Dhond, et al., 1989) (Brown, et al., 1992) (Jones, et al., 1997)(Scharstein \& Szeliski, 2002) assume that images have been rectified so that the task is simplified to establishing correspondences between corresponding rows of the rectified images. The standard process for rectification is homography based, also called planar rectification, where image planes are transformed so that the corresponding space planes coincide (Ayache \& Hansen, 1998) (Hartley, 1999). A major limitation of this class of 
approaches is that, if epipoles are located in the images, planar rectification produces infinitely large images. This problem has now been solved under specific conditions by using either cylindrical (Roy, et al., 1997) or spherical rectifications (Pollefeys, et al., 1999)(Wan \& Zhou, 2008). In addition to adding significant computational complexity, these methods still overlook issues such as sub pixel coordinates, infinite epipoles (Lim, et al., 2004) and the non preservation of conjugate epipolar polarities (Oram, 2001). An alternative to these approaches is to fully calibrate cameras to estimate the appropriate transformation between images. However, since this process is usually manual or relies on very specific environments, its usage has very strong limitations. In any case, all these methods depend on finding a set of accurate matching points, resampling images and appropriate lens distortion models, of all which affect the matching performance. Moreover, there are scenarios where these methods are totally inadequate. For example, visual tracking is seriously degraded by weather conditions when the presence of water drops produces undesirable temporal and localised distortions (Barnum, et al., 2007) (Garg \& Nayar, 2004); similarly, vision systems on space exploration rovers are affected by dust accumulation (Willson, et al., 2005).

Although performing dense pixel matching without prior image transformation is an attractive proposition, very few algorithms have been suggested. This problem was addressed using either multi-resolution image correlation (Duchaineau, et al., 2007)(Zhengping, 1988) or a genetic algorithm (Tippetts, et al., 2010). It was also suggested that a self-organizing neural network could potentially be used under these conditions (Vanetti, et al., 2009). Unfortunately, all these methods display high computational complexity and recursivity, which makes them unsuitable for real-time and hardware implementations.

This review highlights the limitations of rectification and calibration procedures and shows that, currently, bypassing this step leads to solutions with high computation costs. In order to address this, a novel algorithm could be designed by extending one of the approaches developed to tackle in realtime the simpler task of scanline matching (Forstmann, et al., 2004) (Gong, et al., 2006) (Lu, et al., 2009) (Salmen, et al., 2009) (Wang, et al., 2006) (Yang, et al., 2006). Among them, techniques based on DP seem particularly suitable: although they are not the most accurate, performance analysis has shown they provide an excellent compromise between accuracy and speed (Cox, et al., 1996)
(MacLean, et al., 2010) (Scharstein \& Szeliski, 2002) (Brown, et al., 2003) (Tappen \& Freeman, 2003). Their low intrinsic computational complexity even led to a recent FPGA hardware implementation (MacLean, et al., 2010).

In this paper, we propose a novel dense pixel matching algorithm based on the DP paradigm which can operate with unrectified images without camera calibration.

\section{ALGORITHM}

We propose a novel dynamic programming algorithm which is able to establish dense pixel correspondences between two unrectified and/or distorted images. Since our method is based on a DP approach, it relies on two main steps: the storage of local calculations and their reuse to produce a global solution. In this section, we first describe how a 3D scoring matrix is computed. Then, we propose a refinement of these calculations, i.e. introduction of the extended gap concept. Finally, we detail the backtracking phase which allows generating optimal global correspondences.

It is important to note that, although the usage of 3D matrices within DP based pixel matching algorithms was proposed by Sun (Sun, 2002) (Sun-2, 2002), this was only a means of efficiently calculate the correlations over a sliding window. This did not address the requirement of image rectification and was only suitable for scanline matching.

\subsection{D Scoring Matrix}

Similarly to many dynamic programming-based algorithms designed for scanline matching (Geiger, et al., 1992) (Belhumeur, 1996) (Cox, et al., 1996) (Torr \& Criminisi, 2004), the first stage of our algorithm fills in a scoring matrix according to a given scoring scheme. However, our approach does not restrict itself to finding pixel correspondences between scanlines, but between a scanline and an entire image. The strength of this scheme is that images do not need to be rectified and matching can be achieved even when one of the images displays important distortions. Consequently, a scanline on the first image does not need to match a straight line, but may correspond to a curve in the second image (see Figure 1). Since this algorithm is an extension of an algorithm designed for matching stereo pairs of images (Authors, 1111), we use the same convention: the first and second images are referred as the left and right images. 


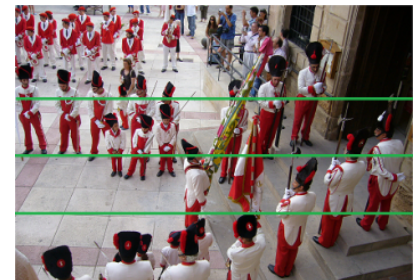

a)

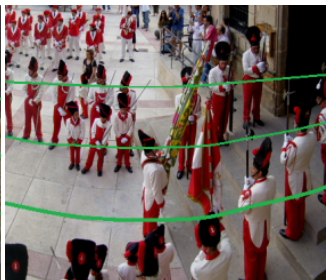

b)
Figure 1: Views of the same scene captured using different camera lenses. Green curves: groundtruth correspondence between 3 scanlines of image a) and pixels of image b).

First, a 3D scoring matrix is generated for each scanline $s$ of the left image. Assuming the left image scanlines have a length $\mathrm{L}$ and the dimensions of the right image are $\mathrm{WxH}$ (width $\mathrm{x}$ height), the size of the scoring matrix, $s c$, is $\mathrm{LxWxH}$. A matrix cell $c i, j, k$ is defined by three coordinates, where $i$ represents the elements of the scanline and $j$ and $k$ the column and line of the right image (see Figure 2).

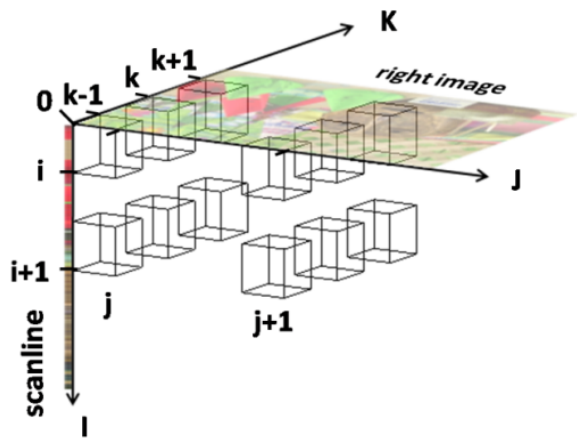

Figure2: 3D scoring matrix.

Matrix cells store the maximum value which can be achieved by extending a previous alignment. When a DP approach is used to find correspondences between two scanlines, an alignment can only be extended in three manners: pixels of the left and right images may have similar values, i.e. there is a match, or one of the pixels may be occluded in either the first or the second sequence, i.e. a gap has to be introduced. Here, an alignment finishing in the cell, $c i, j, k$, can be extended in seven ways.

Since a match means that a pixel of the scanline corresponds to a pixel in the image, the matching cell must be contiguous to the previous cell, $c_{i, j, k}$. Moreover, dynamic programming approach requests moving forward in order to come to an end. Consequently, a match can occur in either cell

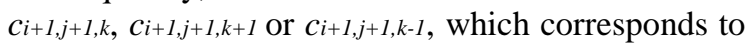
a correspondence between the next pixel in the scanline $(i+1)$, and respectively, the next pixel in the next column $(j+1)$ in the same line $(k)$, match $m_{1}$, the line below $(k+1)$, match $m 2$, or the line above $(k-1)$, match $m_{3}$ (see Figure 3).

Since a change of line and column means moving by a distance of $\sqrt{2}$ pixels in the image, this implicitly adds a virtual gap of $(\sqrt{2}-1)$ pixels. Therefore, this type of match should be less rewarded than a match along the $i+1, j+1, k$ direction.

If matches between pixels cannot be found, a gap has to be introduced in either the image or the scanline. A gap in the image indicates that a scanline pixel does not have any correspondence in the image: this pixel is occluded in the right image. Consequently, the alignment is extended by moving to the next element in the scanline, i.e. $c_{i+1, j, k}$ (gap g4) (see Figure 4).

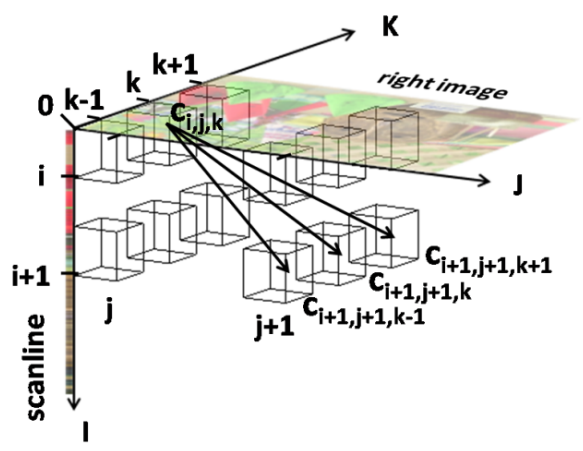

Figure 3: Matching.

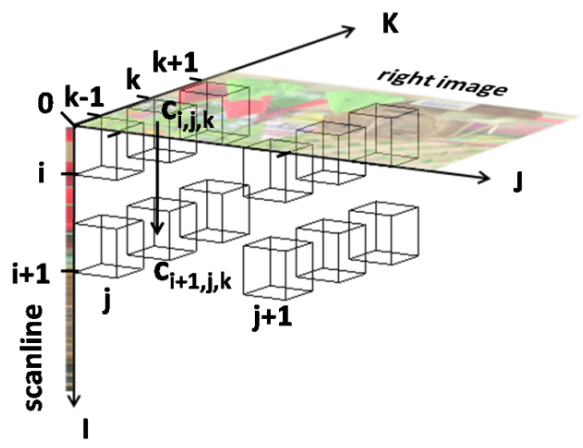

Figure 4: Gap in the image.

Alternatively, image pixels may be occluded in the left image. This implies not moving in the $I$ direction, i.e. the displacement in the matrix is towards either $c_{i, j+l, k}$ (gap g1), $c_{i, j+l, k+l}$ (gap g2) or $c_{i, j+l, k-1}$ (gap $g 3$ ) (see Figure 5). Similarly to the case of matching, a change of line implies adding an extra gap of $(\sqrt{2}-1)$ pixels, which also needs to be penalised.

As shown, each cell of the 3D scoring matrix can be accessed from 7 directions. During the filling process, for each of these directions the cell value is 
calculated according to the cost of the move, i.e. match or gap costs: the highest value and the direction(s) it is coming from are stored in the cell. This information is used in the backtracking process.

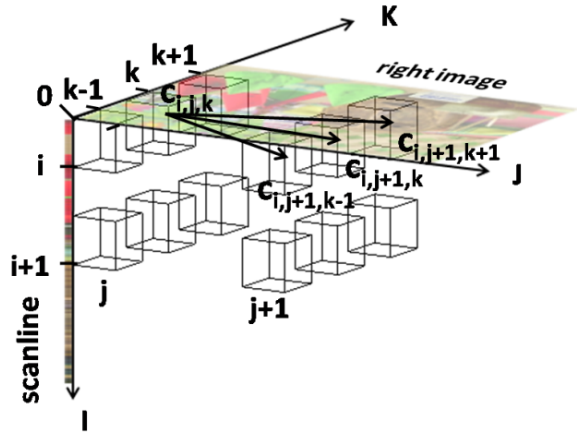

Figure 5: Gap in the scanline.

In order to define the costs of these possible moves, we introduce 3 parameters: $m$ is the reward for a perfect match, $g$ is the penalty for a single gap, and $\Delta$ is the penalty for an imperfect match, which is set at the absolute value of the intensity difference between the scanline pixel, $s(i)$, and the image pixel, $\operatorname{Im}(j, k)$.

$$
\Delta=|s(i)-\operatorname{Im}(j, k)|
$$

Using these parameters, the cost of adding an extra gap of $(\sqrt{2}-1)$ pixels, $p$, can be expressed by:

$$
p=|(\sqrt{2}-1)(m-g)|
$$

Before starting the filling process, the matrix needs to be initialised. First, the initial alignment score is set to zero. This involves extending the initial matrix of dimensions $\mathrm{LxWxH}$ with the planes $(0, J, K)$ and $(I, 0, K)$. Since the alignment can start from any line of the image, cells with coordinates $i=0$ and $j=0$ are filled with zeros. Then other cells from the planes $(0, J, K)$ and $(I, O, K)$ are initialised according to cumulated gap penalties.

Finally, the scoring matrix, $s c$, is filled in according to the following pseudo-code:

$$
\begin{aligned}
& \text { for } i=1 \text { to } L \\
& \text { for } j=1 \text { to } W \\
& \text { for } \mathrm{k}=1 \text { to } H \\
& \Delta \leftarrow|s(i)-\operatorname{Im}(j, k)| \\
& \mathrm{m} 1 \leftarrow \mathrm{sc}(i-1, j-1, \mathrm{k})+\mathrm{m}-\Delta \\
& \mathrm{m} 2 \leftarrow \mathrm{sc}(i-1, j-1, \mathrm{k}+1)+\mathrm{m}-\Delta-\mathrm{p} \\
& \mathrm{m} 3 \leftarrow \mathrm{sc}(i-1, j-1, \mathrm{k}-1)+\mathrm{m}-\Delta-\mathrm{p} \\
& \mathrm{g} 1 \leftarrow \mathrm{sc}(i, j-1, \mathrm{k})+\mathrm{m}-\mathrm{g} \\
& \mathrm{g} 2 \leftarrow \mathrm{sc}(i, j-1, \mathrm{k}+1)+\mathrm{m}-\mathrm{g}-\mathrm{p} \\
& \mathrm{g} 3 \leftarrow \mathrm{sc}(i, j-1, \mathrm{k}-1)+\mathrm{m}-\mathrm{g}-\mathrm{p} \\
& \mathrm{g} 4 \leftarrow \mathrm{sc}(i-1, j, \mathrm{k})+\mathrm{m}-\mathrm{g} \\
& \mathrm{sc}(i, j, \mathrm{k}) \leftarrow \max (\mathrm{m} 1, \mathrm{~m} 2, \mathrm{~m} 3, \mathrm{~g} 1, \mathrm{~g} 2, \mathrm{~g} 3, \\
& \text { end for } \\
& \text { end for } \\
& \text { end for }
\end{aligned}
$$

As a consequence, the time complexity of the proposed algorithm is $O(L W H)$ per scanline.

\subsection{Extended Gap}

In the initial scoring scheme, each individual gap has a fixed penalty. However, due to the nature of stereo images and successive video frames where different perspectives create partial object occlusions, occluded pixels tend to cluster instead of being equally distributed across an image. We propose to exploit this observation by introducing a lower penalty for new gaps which extend existing gaps. An affine gap penalty is used where the initial gap opening penalty is set at $g$ and each extension of a gap increases the total penalty by a lower value, $e$, $(e<g)$. Consequently, a gap of length $l$ will only encounter a penalty of $g+(l-1) e$ in this new scheme.

\subsection{Backtracking}

Once the 3D scoring matrix has been filled, global alignment(s) between the scanline and the image are recovered by backtracking. This is achieved, first, by retrieving in the matrix cell with the highest score obtained for a global alignment. Then, from that cell, using the stored direction information, one or more paths are reconstructed to the origin of the matrix and converted into alignments.

Global alignment scores are found in four planes of the matrix (see Figure 6). If the last pixel of the scanline corresponds to a pixel of the image, global scores are recorded in the plane $i=L$ (orange plane, Figure 4). Alternatively, the end of the scanline may not have any correspondence in the image because it belongs to a non overlapping region. As a consequence, the last scanline pixel with an image pixel match would be located on one of the borders of the image. This score could be read in either the plane $k=1$ (yellow plane, top of the image), the plane $k=H$ (green plane, bottom of the image) or the plane $j=W$ (purple plane, right of the image).

From the cell(s) with the highest score within these four planes, global alignment are discovered by backtracking inside the matrix using the stored information regarding the direction(s) the score of the cell of interest is coming from. Since a cell score comes from the highest value among seven directions, two or more directions could lead to equal maximum scores. Consequently, during backtracking several optimal alignments could be produced. Although strategies, such as choosing the solution which is the most consistent with this of the 
previous scanline, can be used to adopt the most likely path, in this work, a unique alignment is generated by selecting at random one of the global solutions.

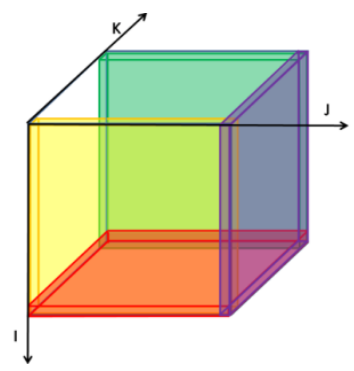

Figure 6: Location of global alignment scores.

\section{EXPERIMENTAL RESULTS}

In order to validate the proposed algorithm a set of experiments were conducted. First, the algorithm is evaluated using a standard stereo matching evaluation framework using rectified images. Then, the potential of our approach is demonstrated by processing unrectified and non-linearly distorted images.

Although parameters are optimised for each stereo pair using the rectified image scenario, typical values are $\mathrm{m}=256, \mathrm{~g}=181$ and $\mathrm{e}=156$. Since the processing of disparity maps by a median filter increases accuracy by enforcing some inter-scanline continuity (Veksler, 2005) (Deng \& Lin, 2006), in some experiments, we apply this filter in a postprocessing step.

\subsection{Rectified Images}

Our algorithm was first evaluated using the Middlebury Stereo Evaluation framework (Scharstein \& Szeliski, 2003) which is widely used as a benchmark tool by the computer vision community when assessing performance of matching algorithms between rectified images.

Initially, we validate our DP implementation by disabling the ability of changing lines in the scoring matrix. Therefore, it takes advantage of the fact that images are known to be rectified. Table 1 shows that, once a median filter is applied onto the raw disparity maps, this basic version of the proposed algorithm (Rectified), that was introduced in a previous paper (Dieny, 2011), performs similarly to Bobbick et al. (Bobick \& Intille, 1999), which is the reference among standard DP approaches. Performances of the proposed algorithm (Unrectified) are also provided in Table 1. Although the ability to change lines degrades significantly stereo matching accuracy, the quality of produced disparity maps remains reasonable (Figure 7). In the next section, we show that this performance reduction in the case of rectified images is rewarded by the ability to process more complex sets of image pairs.
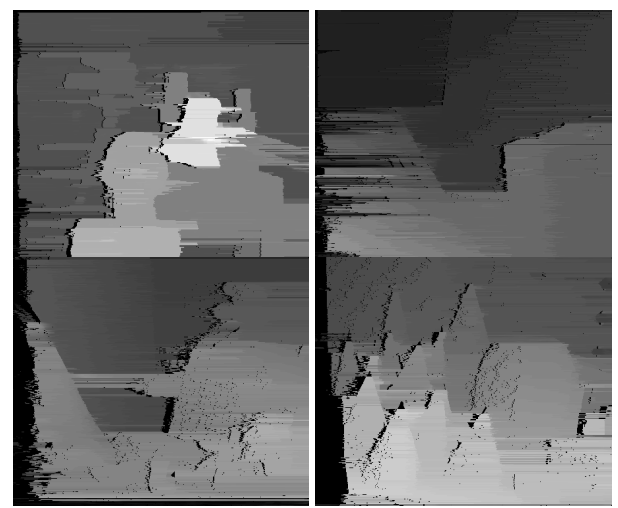

Figure 7: Raw disparity maps obtained for Middlebury dataset (Unrectified).

Table 1: Performance comparison using the Middlebury Stereo Evaluation framework.

\begin{tabular}{|c|c|c|c|c|c|}
\hline$\%$ & $\begin{array}{l}\text { Tsukuba } \\
\text { (non occ) }\end{array}$ & $\begin{array}{c}\text { Venus } \\
\text { (non occ) }\end{array}$ & $\begin{array}{c}\text { Teddy } \\
\text { (non occ) }\end{array}$ & $\begin{array}{l}\text { Cones } \\
\text { (non occ) }\end{array}$ & $\begin{array}{c}\text { Average } \\
\text { (bad pixels) }\end{array}$ \\
\hline $\begin{array}{c}\text { Bobbick } \\
\text { et al. 99 }\end{array}$ & 4.12 & 10.1 & 14.0 & 10.5 & 14.2 \\
\hline Rect. & 6.74 & 10.7 & 14.1 & 11.0 & 16.7 \\
\hline Rect $^{*}$ & 4.63 & 7.40 & 10.7 & 7.75 & 13.4 \\
\hline $\begin{array}{c}\text { Un- } \\
\text { rect. }\end{array}$ & 11.0 & 18.6 & 28.2 & 23.9 & 28.5 \\
\hline $\begin{array}{c}\text { Un- } \\
\text { rect }\end{array}$ & 9.47 & 16.7 & 26.3 & 21.6 & 26.5 \\
\hline
\end{tabular}

*: applying a Median Filter

\subsection{Unrectified Images}

The proposed algorithm has a unique capacity to process unrectified and distorted images. This is first demonstrated by applying rotations to the right images of the Middlebury dataset (Scharstein 20022003) to simulate unrectified images so that (see Figure 8) quantitative results can be produced for evaluation.

Since by design, the algorithm can only change line by going either on the next pixel above or below the current pixel, motions across an image are limited to angles comprised in the interval $\left[-45^{\circ}, 45^{\circ}\right]$ from the $\mathrm{x}$ axis. Consequently, an image rotation by an angle outside that range cannot be directly solved. 
However, this situation can be handled by using the strategy described at the end of the section.

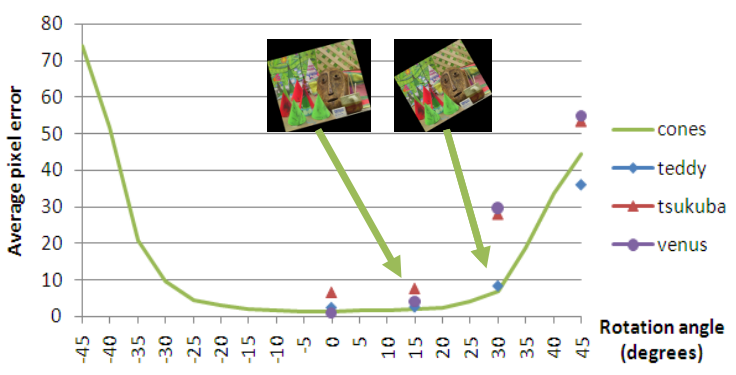

Figure 8: Raw Average pixel error for non occluded pixels as a function of image rotation.

In Figure 8, average pixel error is depicted according to the angle the right image was rotated. Data are provided for the 'Cone' image with a $5^{\circ}$ resolution within the $\left[-45^{\circ}, 45^{\circ}\right]$ range, whereas the other images are rotated with a $15^{\circ}$ resolution within the $\left[0^{\circ}, 45^{\circ}\right]$ range. Since the metrics employed in the automatic evaluation framework are not publicly available, error values reported in Figure 6 cannot be directly compared to those shown in Table 1. Here, we define average pixel error as the average difference between the estimated and actual disparity maps. Note that occluded pixels in either map are not considered.

Consistent results are obtained for all the images showing good performance for rotations within the $\left[-20^{\circ}, 20^{\circ}\right]$ range. Outside this range, pixel errors increase significantly. This behaviour reveals some weakness in the current penalty scheme used when a change of line occurs, Eq. (2). When rotations by an angle lower than $22.5^{\circ}$ are applied, the scoring matrix contains a majority of scanline moves and consecutive line jumps are rare. However, in the case of larger rotations, score inaccuracies introduced by frequent line change affect significantly the calculations of the scoring matrix which leads to global errors in pixel correspondences.

Although, by design, it is not possible to solve alignments between images that have been rotated by more than $45^{\circ}$, the framework can be easily extended to tackle these situations. The strategy relies on repeating the pixel correspondence process after introducing artificial rotations. The original right image is rotated 8 times by an angle of $45^{\circ}$ so that one of these 8 images will fall within the [$\left.45^{\circ}, 45^{\circ}\right]$ interval when considering the combined rotation, i.e. original plus artificial. Each of the 8 sets of correspondences is associated with an average matching score calculated from maxima found in the scoring matrices. These alignment scores are them used to identify the meaningful disparity map.

Figure 9 illustrates this with an example, where the 'cones' right image was rotated by $60^{\circ}$. Analysis of the curve shows that the best alignment score is obtained when an artificial rotation of $-45^{\circ}$ is applied: this corresponds to an actual combined rotation of $15^{\circ}$, which can be successfully processed by the proposed algorithm. This approach could also be used to address the drop of performance caused by images which were subjected to an original rotation above $22.5^{\circ}$. In this application, artificial rotations of $22.5^{\circ}$ or lower would be required.

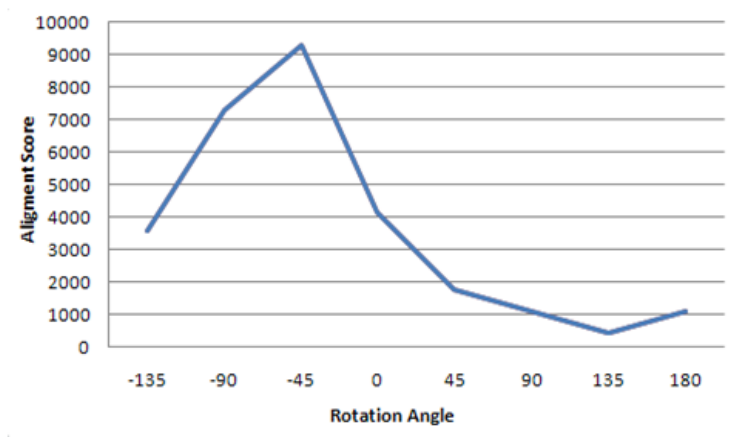

Figure 9: Alignment scores obtained after adding an artificial rotation (x axis).

\subsection{Distorted Images}

Given the general nature of our algorithm, its usage is not limited to finding pixel correspondences between unrectified images, but it can be applied to images affected by non-linear distortions.

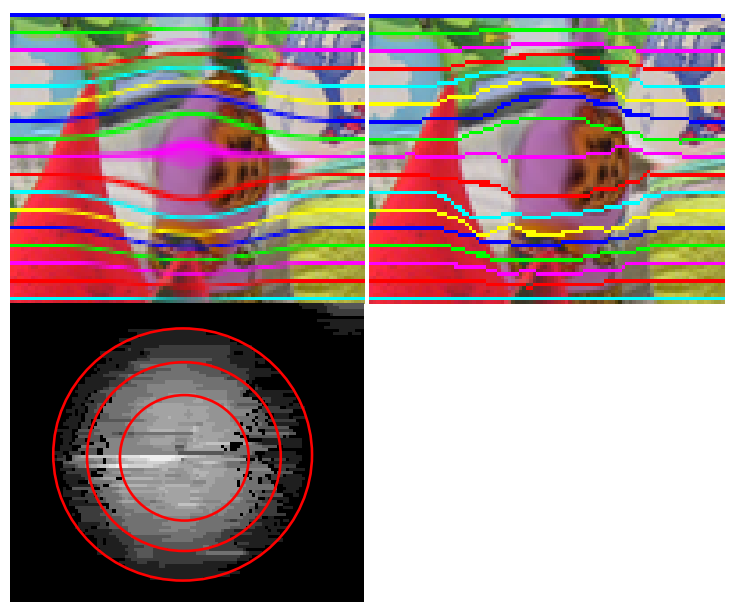

Figure 10: Correspondences in a distorted area: a) actual, b) estimated c) disparity map 
In order to illustrate this, three experiments were conducted. First, water drops on a camera lens were simulated by applying lenticular distortions to the test image (Figure 13a,b). Then correspondences between the original and the distorted images were calculated. Figures 13c) and 10a,b) show that our algorithm detects the distortions and find good correspondences by seeking optimal alignment within the image. The associated disparity maps Figures 13b) and 10c) reveal our method identifies not only distortion areas, but also their lenticular nature, since disparity values tend to be higher in the centres and radially decrease.

In the second experiment, a scene was captured using different camera lenses (see Figure 1). The second lens introduces a notable spherical aberration (see Figure 1b) but also a small change of scale. Figure 11 shows the result of matching three scanlines of the first image (Figure 1a) against the whole second image (Figure 1b). The central line and two parallel lines at 60 pixels from the centre were chosen to show the lens aberration and the performance of our method under those conditions. The proposed methodology allows us to find a reasonable correspondence in spite of the distortion.

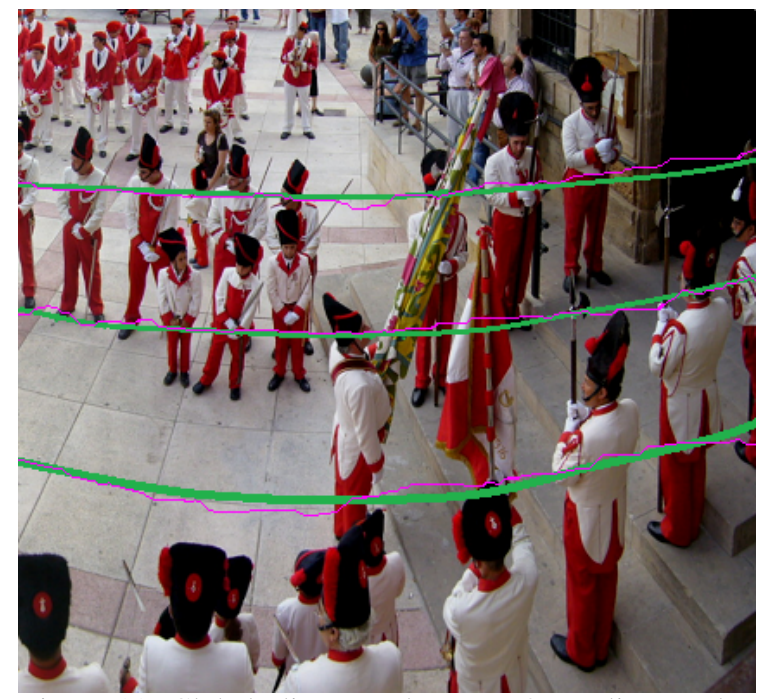

Figure 11: Global alignment between 3 scanlines and a whole image in case of distortion. Green lines represents the groundtruth while purple curves are calculated by our algorithm.

Finally, in the third experiment, we processed the picture of a standing lady and its retouched version (Figures 12a,b), which shows a streamlined body. This digital intervention is clearly identified on Figure $12 \mathrm{c}$ and d) where the left and right parts of the different limbs generally appear to have been moved in opposite directions. In addition, dark patches on Figure 12d highlights areas where a cloning tool was applied to reconstruct missing background.
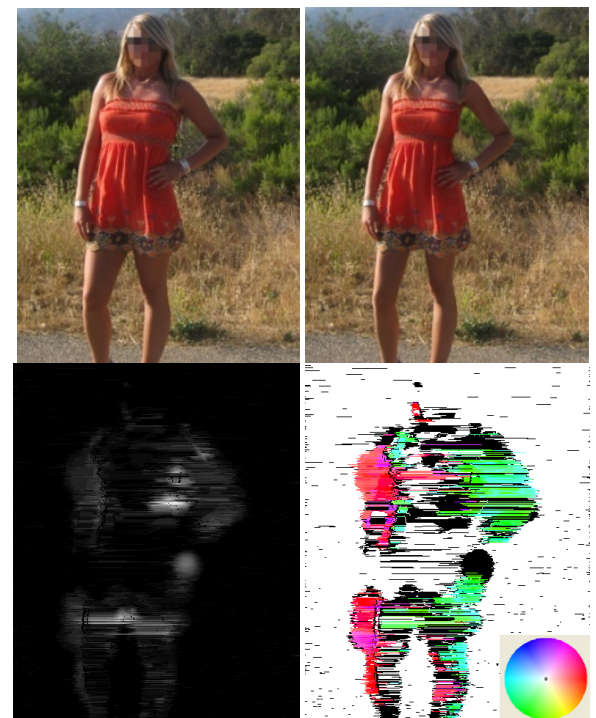

Figure 12: Character a) before and b) after retouching. Disparity maps c) Standard and d) using a colour palette (enclosed) to highlight disparity directions.

\section{CONCLUSIONS}

In this paper, we introduce a novel algorithm for dense pixel matching between unrectified images without any pre-processing stage. Based on a dynamic programming approach, our main contribution is the design of a 3D scoring matrix which allows finding the best correspondence between a line and a whole image. As demonstrated in experiments, good alignments are obtained using images rotated by up to $20^{\circ}$ and non-linearly distorted. In addition, its structure, which relies on a single DP process, makes it suitable for hardware implementation.

As future work, we propose to improve the current scoring scheme and exploit inter-scanline information to improve the algorithm performance. Thus, for instance, the usage of uniqueness regarding the scanline matching would allow accelerating the processing by excluding part of the $3 \mathrm{D}$ cube.

\section{REFERENCES}

Ayache, N., Hansen, C., 1988. Rectification of images for binocular and trinocular stereovision. In International Conference on Pattern Recognition, pp. 11-16. 


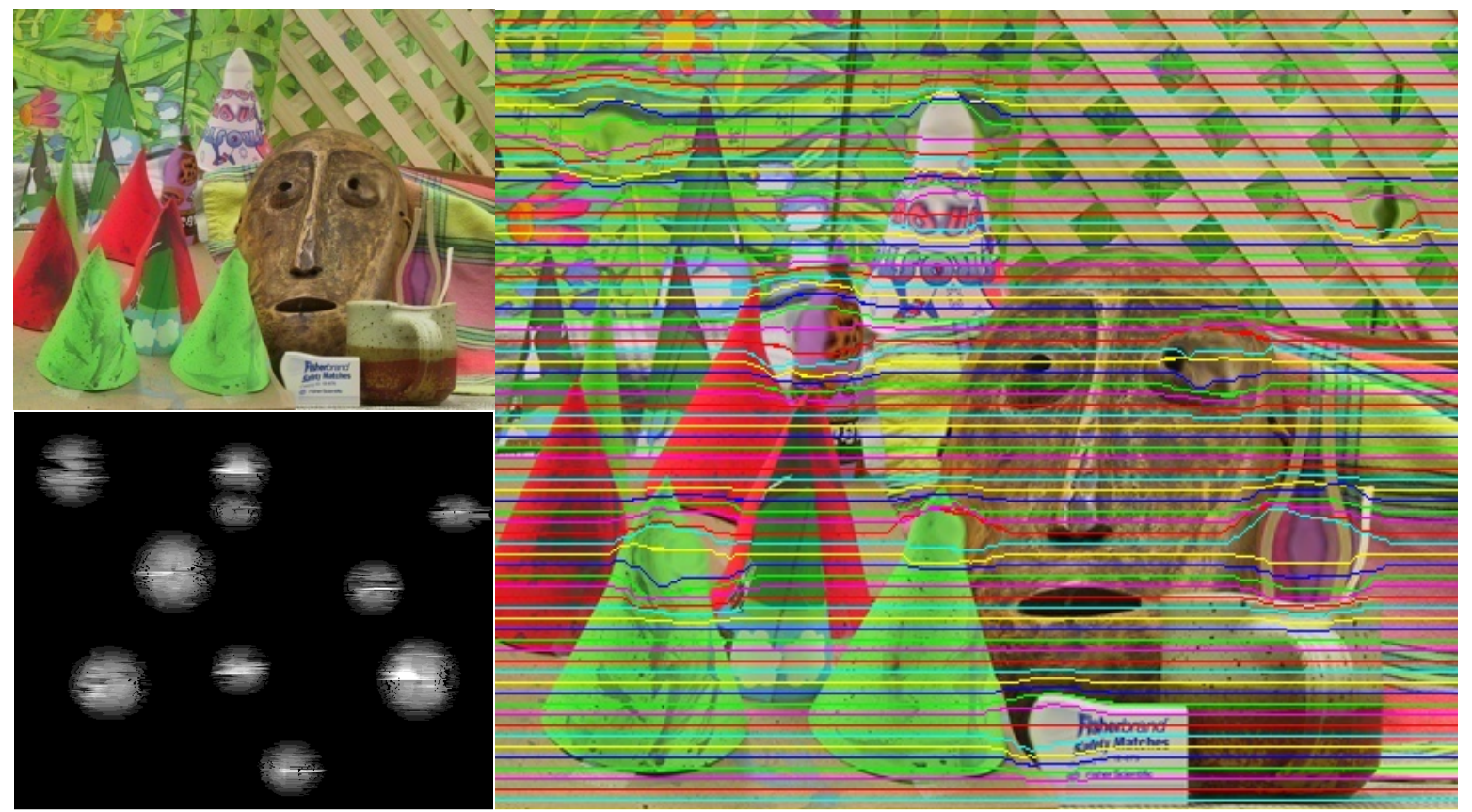

Figure 13: Top left: a) Distorted image. Bottom Left: b) Disparity map between distorted and original images. Right: c) Estimated correspondences.

Baker, H., Binford, T., 1981. Depth from edge and intensity based stereo. In IJCAI81, pp.631-636.

Barnard, S.T., Fischler, M.A., 1982. Computational stereo. In ACM Comp. Surveys, 14(4), pp.553-572.

Barnum, P., Kanade, T., Narasimhan., S.G., 2007. SpatioTemporal Frequency Analysis for Removing Rain and Snow from Videos. In $P A C V$.

Belhumeur, P.N., 1996. A Bayesian approach to binocular stereopsis. International Journal of Computer Vision, 19(3), pp.237-260.

Bobick, A.F., Intille, S.S., 1999. Large occlusion stereo. International Journal of Computer Vision, 33(3), pp.181-200.

Brown., L.G., 1992. A survey of image registration techniques. In ACM Comp. Surveys, 24(4), pp.325376.

Brown, M.Z., Burschka, D., Hager, G.D., 2003. Advances in computational stereo, IEEE Transactions on Pattern Analysis and Machine Intelligence, 5 (8), pp. 9931008.

Cox, I.J., Hingorani, S.L., Rao, S.B., Maggs, B. M., 1996. A maximum likelihood stereo algorithm. Computer Vision and Image Understanding, 63(3), pp.542-567.

Deng, Y., Lin, X., 2006. A fast line segment based dense stereo algorithm using tree dynamic programming. In European Conference on Computer Vision, Graz, Austria, May 7 - 13.

Dhond, U.R., Aggarwal, J.K., 1989. Structure from stereo: a review. In IEEE Trans. on Systems,Man, and Cybern., 19(6), pp.1489-1510.
Dieny, R., Thevenon, J., Martinez-del-Rincon, J., Nebel, J.C., 2011. Bioinformatics inspired algorithm for stereo correspondence, In VISAPP, Vilamoura, Portugal, March 5-7.

Duchaineau, M., Cohen, J., Vaidya, S., 2007. Toward Fast Computation of Dense Image Correspondence on the GPU. In High Performance Embedded Computing Workshop, Lincoln Laboratory, Massachusetts Institute of Technology, pp.91-92.Forstmann.

Forstmann, S.,Kanou, Y., Ohya, J., Thuering, S., Schmitt, A., 2004. Real-Time Stereo by using Dynamic Programming, In Computer Vision and Pattern Recognition Workshop, Washington, DC, USA, 27 June-2 July 2004.

Garg, K., Nayar, S. K., 2004. Detection and removal of rain from videos. In CVPR.

Geiger, D., Ladendorf, B., Yuille, A., 1992. Occlusions and binocular stereo. In European Conference on Computer Vision, pp.425-433.

Gong, M., 2006. Enforcing Temporal Consistency in RealTime Stereo Estimation. In ECCV 2006, Part III, LNCS 3953, pp.564- 577, 2006.

Hartley, R.I., 1999. Theory and practice of projective rectification. International Journal of Computer Vision, 35(2), pp.115-127.

Jones. G.A., 1997. Constraint, Optimization, and Hierarchy: Reviewing Stereoscopic Correspondence of Complex Features. In Computer Vision and Image Understanding, 65(1), pp. 57-78.

Lazaros, N., Sirakoulis, G.C., Gasteratos A., 2008. Review of Stereo Vision Algorithms: From Software to 
Hardware. International Journal of Optomechatronics, 2(4), pp.435 - 462.

Lim, S.N., Mittal, A., Davis, L., Paragios, N., 2004. Uncalibrated stereo rectification for automatic 3D surveillance. In International Conference on Image Processing, 2, pp.1357.

Lu, J., Ke Zhang, Lafruit, G., Catthoor, F. 2009. Real-time stereo matching: A cross-based local approach. In International Conference on Acoustics, Speech and Signal Processing, pp.733-736, Washington, DC, USA, April 19 - 24, 2009.

MacLean, W.J., Sabihuddin, S., Islam, J., 2010. Leveraging cost matrix structure for hardware implementation of stereo disparity computation using dynamic programming. Computer Vision and Image Understanding, In Press.

Needleman, S.B., Wunsch, C.D., 1970. A general method applicable to the search for similarities in the aminoacid sequence of two proteins. Journal of Molecular Biology, 48(3), pp.443-53.

Note, J.B., Shand, M., Vuillemin, J., 2006. Real-Time Video Pixel Matching. In FPL, pp. 1-6.

Ohta, Y., Kanade, T., 1985. Stereo by intra- and interscanline search using dynamic programming. IEEE TPAMI, 7(2), pp.139-154.

Oram., D., 2001. Rectification for Any Epipolar Geometry. In $B M V C$, pp. 653-662.

Pollefeys, M., Koch, R., Van Gool, L., 1999. A simple and efficient rectification method for general motion. In International Conference on Computer Vision, vol 1, pp. 496-501.

Roy, S., Meunier, J., Cox, I., 1997. Cylindrical Rectification to Minimize Epipolar Distortion. In Conference on Computer Vision and Pattern Recognition, pp.393-399.

Salmen, J., Schlipsing, M., Edelbrunner, J., Hegemann, S., Lueke, S., 2009. Real-time stereo vision: making more out of dynamic programming. In International Conference on Computer Analysis of Images and Patterns, Münster, Germany, Sept. 2-4.

Scharstein, D., Szeliski, R, 2002. A taxonomy and evaluation of dense two-frame stereo correspondence algorithms. International Journal of Computer Vision, 47(1), pp.7-42.

Scharstein, D., Szeliski, R, 2003. High-accuracy stereo depth maps using structured light. In IEEE Computer Society Conference on Computer Vision and Pattern Recognition, vol. 1, pp. 195-202.

Sun, C., 2002. Fast Stereo Matching Using Rectangular Subregioning and 3D Maximum-Surface Techniques.
In International Journal of Computer Vision archive, 47 (1-3), pp. 99-107.

Sun., C., 2002. Fast Optical Flow Using 3D Shortest Path Techniques. In Image and Vision Computing, 20(13/14), pp. 981-991.

Tappen, M., Freeman, W., 2003. Comparison of graph cuts with belief propagation for stereo, using identical MRF parameters, in: IEEE International Conference on Computer Vision (ICCV), 2, pp. 900-906.

Tippetts, B., Lee, D.J., Archibald, J., 2010. Fast correspondence of unrectified stereo images using genetic algorithm and spline representation. In Intelligent Robots and Computer Vision XXVII: Algorithms and Techniques, 7539, 17 January 2010.

Torr, P.H.S., Criminisi, A., 2004. Dense stereo using pivoted dynamic programming. Image and Vision Computing, 22(10), pp.795-806.

Vanetti, M., Gallo, I., Binaghi, E., 2009. Dense TwoFrame Stereo Correspondence by Self-organizing Neural Network. In ICIAP 2009, LNCS 5716, pp.1035-1042.

Veksler, O., 2005. Stereo correspondence by dynamic programming on a tree. In Computer Vision and Pattern Recognition, San Diego, CA, USA, 20-26.

Willson, R.G., Johnson, A.E., Goguen, J.D., 2005. MOC2DIMES: A camera simulator for the mars exploration Rover descent image motion estimation system. In Proc. 8th Int'l. Symp. Artificial Intelligence, Robotics and Automation in Space.

Wan, D., Zhou, J., 2008. Stereo vision using two PTZ cameras. Computer Vision and Image Understanding, 112, pp.184-194.

Wang, L., Liao, M., Gong, M., Yang, R., Nistér, D., 2006. High-quality real-time stereo using adaptive cost aggregation and dynamic programming. In $3 D$ Data Processing, Visualization and Transmission. Chapel Hill, USA, June 14-16.

Yang, Q., Wang, L., Yang, R., 2006. Real-time Global Stereo Matching Using Hierarchical Belief propagation. In BMVC 2006, Edinburgh, UK.

Yaguchi, Y., Iseki, K., Oka, R., 2009. Optimal Pixel Matching between Images. In PSIVT pp. 597-610.

Yin, X.C., Sun, J., 2007. Perspective Rectification for Mobile Phone Camera-Based Documents Using a Hybrid Approach to Vanishing Point Detection, CBDAR'07, pp. 37-44.

Zhengping, J., 1988. On the multi-scale iconic representation for low-level computer vision systems. In $P h D$ thesis, The Turing Institute and The University of Strathclyde, 1988. 